\title{
No evidence for apparent extent between parallels as the basis of the Poggendorff effect
}

\author{
R. H. DAY, W. J. JOLLY, and F. M. DUFFY \\ Monash University, Melbourne, Australia
}

\begin{abstract}
Data from five experiments consistently failed to support an explanation of the Poggendorff misalignment and misbisection effects in terms of underestimation of extent between parallel lines. Although underestimation of vertical extent between parallel lines was confirmed, the effects on it of the kind and number of elements on the parallels were opposite to their effects on apparent misalignment and misbisection. Furthermore, right-angle extent between oblique parallels was overestimated. When vertical extent between oblique parallels and oblique extent between vertical parallels (as in the conventional figure) ended in transversals, underestimation was eliminated. It is concluded that the illusions of extent and alignment (or bisection) are independent effects. Both are held to be due to a process of perceptual compromise.
\end{abstract}

Two versions of the Poggendorff figure can be seen in Figure 1. Figure 1A, in which the collinear verticals appear to be misaligned, is merely a rotation of the original figure so that the parallels are oblique and the transversals upright. Figure 1B is Tolansky's (1964) version; if the upper vertical were extended downward it would exactly bisect the space between the two lower verticals but appears as if it would not do so. The direction of apparent misalignment in Figure 1A and apparent "misbisection" in Figure 1B is the same; the upper vertical appears too far to the upper left for both alignment and exact bisection.

Figures $1 \mathrm{~A}$ and $1 \mathrm{~B}$ and a number of their variants were included in two recent series of experiments (Day \& Kasperczyk, 1985; Day, Watson, \& Jolly, 1986). Some of the variants are shown in Figures $1 \mathrm{C}-1 \mathrm{~F}$. In the first series, subjects were required to position a line or dot on the upper oblique parallel so that it appeared to fall either in the same vertical axis as a line or dot on the lower parallel or in the axis of exact vertical bisection of the space between two lines or dots. For all figures, including those with only dots on the parallels (Figures $1 \mathrm{C}$ and 1D), errors, that is, compensations for apparent misalignment and misbisection, were in the same direction and of about the same size. In the second series, it was found that misbisection occurred in figures without the parallels, that is, ones consisting of only three vertical lines (Figure 1E) or three dots (Figure 1F). The effects were also in the same direction and not statistically different from those in figures with parallels (Figures 1B and 1D).

Apparent misalignments or misbisections with dots both with and without oblique parallels and of more or less the same size as those with lines intersecting parallels call

This research was supported by a grant to the first author from the Australian Research Grants Committee. Requests for reprints should be sent to R. H. Day, Department of Psychology, Monash University, Clayton, Victoria 3168, Australia.
A

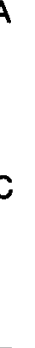

E

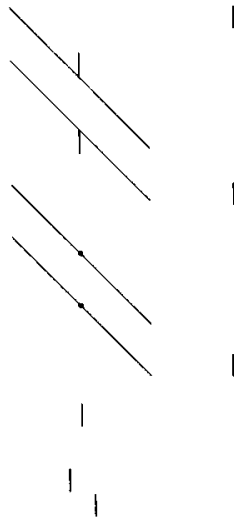

Figure 1. Stimulus figures with and without oblique parallels in which apparent misalignment, that is, apparent displacement of the upper element from the vertical axis of the lower element ( $A$ and C), and apparent misbisection, that is, apparent displacement of the upper element from exact vertical bisection of the space between the two lower elements (B, D, E, and F), are no different with dots or with lines and no different with or without oblique parallels.

into question various explanations of the Poggendorff effect. These include those couched in terms of apparent size of angles, apparent orientation of lines, and depth processing of oblique lines (see Day \& Kasperczyk, 1985). An alternative explanation has therefore been developed. It is argued that in figures like those in Figure 1 alignment or bisections can be made in both the vertical axis and an axis defined or implied by the figure itself. In the case of the figures in Figure 1, the latter axis is at right angles to the parallels. When subjects position a line or dot for apparent alignment or bisection in the vertical, it is contended that they are influenced by its misalignment or misbisection' in the right-angle axis. A compromise therefore occurs which takes the form of a slight shift away from alignment or bisection in the designated vertical axis in the direction of misalignment or misbisection in the nondesignated right-angle axis. The errors 
made in positioning the upper element in Figure 1 are compensations for this compromise.

Wenderoth, O'Connor, and Johnson (1986) have criticized this explanation on the grounds that a perceptual compromise would be unlikely to occur with transversal lines that define the vertical axis. In a follow-up experiment, Wenderoth et al. (1986) did find a difference in vertical bisection between lines and dots when instructions strongly specified vertical bisection and prohibited rightangle bisection. However, both Wenderoth and O'Connor (1987) and Day and Jolly (1987) confirmed the absence of any difference between lines and dots with instructions for apparent vertical bisection. Wenderoth et al. (1986) and Wenderoth and O'Connor (1987) have opted for an explanation of the effect with dots in terms of a confusion between the two axes. Day and Jolly (1987) contend that, as with other perceptual effects, strong "objective" rather than "apparent" instructions encourage cognitive control over the perception of alignment and bisection in figures like those in Figure 1. It is argued that perceptual compromise, a common process in a wide range of situations, is simply prevented from occurring by strongly prohibitive instructions.

Greist-Bousquet and Schiffman (1986) have proposed an alternative explanation of the results reported by Day and Kasperczyk (1985). They argue that apparent misalignment and misbisection in oblique-line figures can be accounted for by underestimation of interparallel extent due to the effect of a Müller-Lyer-type figure between the misaligned elements. This proposal is illustrated in Figure 2. If the parallels in Figure $2 \mathrm{~A}$ were to be moved closer together, as in Figure $2 \mathrm{~B}$, the obliques that are aligned in the former would be misaligned in the latter. Greist-Bousquet and Schiffman (1981a, 1986) contend that apparent interparallel extent is reduced due to the forms of the Müller-Lyer illusion illustrated in Figures $2 \mathrm{C}$ and
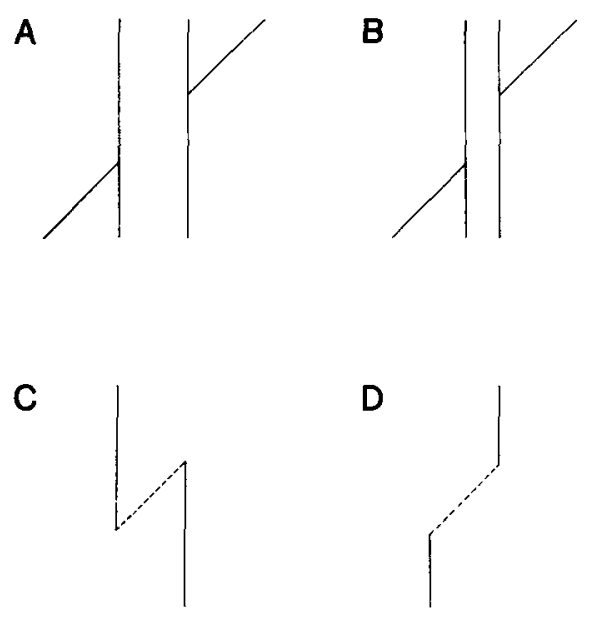

Figure 2. Basis of the explanation of the Poggendorff misalignment effect in terms of apparent underestimation of oblique extent between vertical parallels due to implicit Müller-Lyer figures (see text).
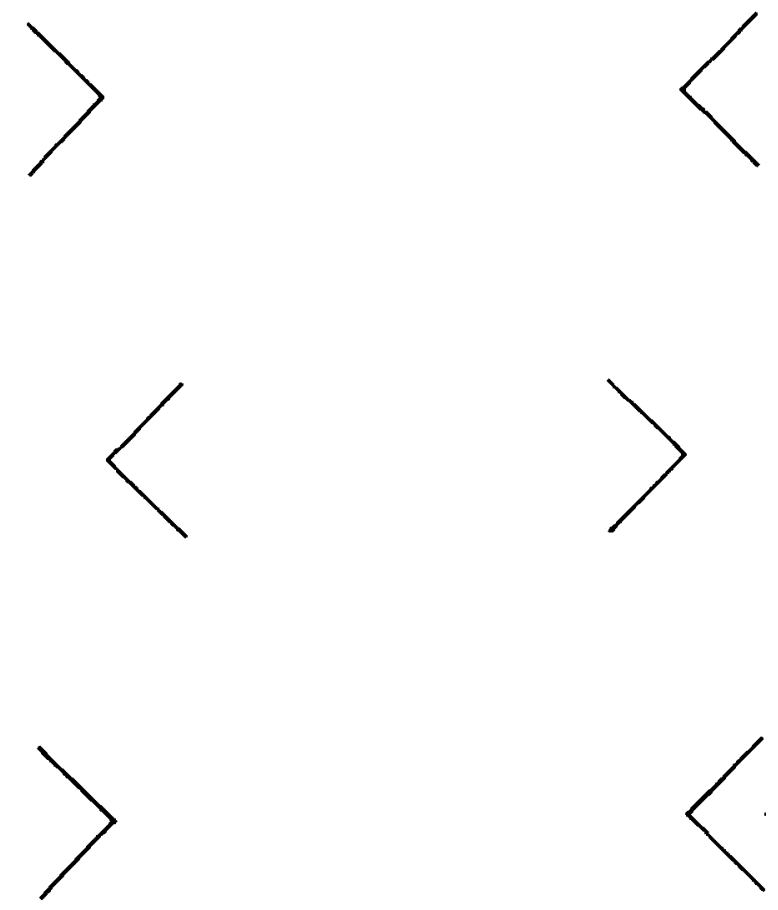

Figure 3. The Morinaga paradox. Horizontal extents between the apexes of the upper and lower pair appear greater than that between the apexes of the central pair. The central apexes appear to be displaced too far inward for alignment with the upper and lower apexes, opposite to that expected in terms of apparent extents.

2D. The Müller-Lyer component in the former could be expected to decrease apparent extent, whereas that in the latter could be expected to increase it. If, as found by Greist-Bousquet and Schiffman (1981b) and Warren and Bashford (1977), the component in Figure $2 \mathrm{C}$ is greater than that in Figure 2D, that is, if underestimation of interparallel extent exceeds overestimation, the net result would be underestimation. That is to say, there would be reduction in the apparent extent between the parallels.

At least three criticisms can be made of this explanation. The first is the assumption that apparent extent due to the Müller-Lyer illusion can determine apparent misalignment or misbisection, that is, that the latter are secondary outcomes of apparent extent. This assumption is seriously questioned by the Morinaga "paradox" (Day, Bellamy, \& Norman, 1983; Morinaga \& Ikeda, 1965; Restle, 1976) shown in Figure 3. Apparent extent between the upper and lower pairs of apexes is markedly greater than for the same physical extent between the central pair. Nevertheless, the apparent vertical misalignment of each group of three apexes is in the opposite direction expected from that of apparent extent; the upper and lower pairs of apexes appear too far inward and the central pair too far outward for vertical alignment (Morinaga \& Ikeda, 1965).

Second, Greist-Bousquet and Schiffman (1981a) clearly demonstrated underestimation of distance between obliquely aligned elements in various forms of the parallel- 
line figure. This is taken to imply that apparent interparallel extent is reduced in the manner illustrated in Figures 2A and 2B. However, underestimation of oblique extent does not by itself imply apparent misalignment. It shows only that the aligned elements appear closer together. It is conceivable that underestimation obliquely does not signify underestimation at right angles to the parallels or in other directions. In any case, Finlay and Caelli (1975) and Wilson and Pressey (1976) found that when oblique extent between vertical parallels was compared with other figural extents it was overestimated. In addition, the former investigators reported that correlations between apparent alignment and apparent extent between parallels were insignificant.

Third, Greist-Bousquet and Schiffman's (1981a) argument turns, in large part, on manipulations of the Poggendorff figure that, while shown to affect apparent extent between obliques, are assumed to affect the size of apparent misalignments. Apparent oblique extent and apparent oblique alignment in the same figure have not been compared.

In light of these considerations, the purpose of the five experiments reported here was to test the validity of the underestimation-of-extent explanation of apparent misalignment in oblique and vertical parallel-line figures. The method for obtaining estimates of apparent extent (making dots on sheets of paper) was essentially and deliberately the same as those used by Greist-Bousquet and Schiffman (1981a). For the first experiment, subjects estimated vertical extent between the oblique parallels, that is, obliquely between the parallels; for the second experiment, they estimated alignment and bisection in the vertical axis. In the third experiment, both vertical extentand that at right angles to the parallels, that is, obliquely and orthogonally between the parallels, were estimated. The fourth and fifth experiments were concerned, respectively, with the generalization of the results of Experiment 3 to the conventional vertically oriented figure and with the effect of intersecting transversals on estimates of extent.

Three frequently used terms should be made clear from the outset. First, since the stimulus figures were placed on a table, the "vertical" interparallel extents to be estimated were not strictly vertical but medial. However, in the interest of uniformity and comparability with earlier studies, the extents are henceforth referred to as vertical. There is no reason to suppose that the illusory displacements in the vertical and medial axes are much different. Second, in two figures in Experiment 2, subjects were required to place a dot so that it appeared to be in the same vertical axis as either a short vertical line or another dot. Although it is not entirely appropriate to refer to the latter as an alignment task (two dots cannot strictly be aligned), that term will nevertheless be used, again in the interest of uniformity. Third, for the sake of brevity, exact vertical bisection is referred to as vertical bisection or simply bisection.

\section{METHODS}

The materials, source of subjects, and general procedure were the same for the five experiments and therefore can conveniently be described first. The stimulus figures are shown in Figures 4 and 5 and are described below along with the composition of subject groups.

\section{Subjects}

Subjects were recruited from among faculty, graduate students, and undergraduates by personal contact and notices.

\section{Method and Procedures}

Subjects estimated interparallel extents (Experiments 1, 3, 4, and 5 ) and alignment or bisection (Experiment 2) in vertical and oblique parallel-line figures by making two dots or one dot, respectively, with a felt-tipped pen on sheets of paper placed on the table at which they sat. The sheets were $15 \times 21 \mathrm{~cm}$ and were placed with one edge flush with the near edge of the table. For seated subjects, the viewing distance to the response sheets was about $45 \mathrm{~cm}$. Thus, $1 \mathrm{~cm}$ at the figure subtended a visual angle of about $1.3^{\circ}$

For estimates of extent, two dots were made so that they appeared to be the same distance apart as clearly designated points on the parallels and in control figures consisting of two vertically or obliquely arranged dots. In Figures $4 \mathrm{~A}$ and $4 \mathrm{~B}$, the designated points were that on the lower parallel midway between the two lower elements and that vertically above it on the upper parallel. In Figures $4 \mathrm{C}$ and $4 \mathrm{D}$, they were the intersection or dot on the lower parallel and a point vertically above them on the upper parallel. In Figures $5 \mathrm{~A}$ and $5 \mathrm{~B}$, the points were indicated by the intersections. For Figure $5 \mathrm{D}$, they were designated as being on the parallels, one vertically above the other. The plain response sheets and the cards of the same size with the stimulus figures were placed side by side on the table with their inner edges touching and in the subject's midline. Four estimates of extent were made for each figure, two with the plain response sheet on the right and the stimulus card on the left and two with these positions reversed. Figures and positions were differently randomized for each subject. Errors in estimation were the differences in millimeters between true and apparent extent to the nearest $.5 \mathrm{~mm}$. Underestimations were treated as negative, and overestimations as positive.

For estimates of vertical alignment and bisection (Experiment 2), one dot was made so that it appeared to be in the same vertical (i.e., medial) axis as a dot or line on the lower parallel (alignment), or in the same vertical axis as a point horizontally midway between two dots or two lines on the lower parallel (bisection). The dots were made on the upper parallel or at about the same distance above the single dot of the control figure. The stimulus figures were photocopied from a master set onto $15 \times 21 \mathrm{~cm}$ sheets. The sheets were placed with either their right or left edge in the subject's midline, that is, in the same positions as the plain response sheets for estimates of extent. Four estimates of alignment or bisection were made for each figure, two with the right edge in the midline and two with the left edge in the midline. Figures and positions were differently randomized for each subject. Errors were the horizontal differences between the points of apparent and exact vertical alignment or bisection measured to the nearest $.5 \mathrm{~mm}$. Errors in the direction to compensate for apparent misalignment or misbisection in Figure 1 were scored as positive; those in the opposite direction were scored as negative.

Instructions emphasized apparent extent and apparent vertical alignment or bisection. Individual scores for estimates of extent for each figure were the means of the four estimates taking sign into account. Likewise, the individual scores for estimates of alignment or bisection for each figure were the means of four estimates also taking sign into account. 


\section{EXPERIMENT 1}

The aim of the first experiment was to determine apparent extent along vertical alignment and bisection axes between oblique parallels.

\section{Method}

There were 12 subjects, 8 women and 4 men.

The four parallel-line stimulus figures are shown in Figure 4. These were drawn about the centers of $23 \times 15 \mathrm{~cm}$ white rectangular cards. The lines were $.5 \mathrm{~mm}$ thick, and the dots were $1.5 \mathrm{~mm}$ in diameter. The $45^{\circ}$ oblique parallels were $80 \mathrm{~mm}$ long and $15 \mathrm{~mm}$ apart at right angles to their length. The vertical extent between the centers of the lines or dots was $22 \mathrm{~mm}$. The vertical lines intersecting the lower parallel in Figures $4 \mathrm{~A}$ and $4 \mathrm{C}$ were $10 \mathrm{~mm}$ long. The distance along the lower parallel between the centers of the two vertical lines and the two dots in Figures $4 \mathrm{~A}$ and $4 \mathrm{~B}$, respectively, was $15 \mathrm{~mm}$. The two-dot control, which is not shown, consisted simply of two $1.5-\mathrm{mm}$ dots arranged one above the other and separated by $22 \mathrm{~mm}$ between centers.

\section{Results and Discussion}

The mean estimate of vertical extent for the two-dot control figure was $-.06 \mathrm{~mm}(S D .90)$. Since this was not significantly different from zero $[t(11)=.24, p>.81]$, and could therefore be regarded as negligible, no subtractions from the scores for the parallel-line figures were made. The mean estimates of vertical extent for the four parallel-line figures are shown with their standard deviations in Table 1. It can be seen that, in Experiment 1, vertical extent was underestimated in all four figures and that underestimates for the two figures with one or two dots on the lower parallel were greater than the two with one or two lines intersecting it. Single-sample $t$ tests showed that the four means were significantly different from zero ( $p<.001$ in all cases). A $2 \times 2$ analysis of variance (ANOVA) in which the main factors were the type of element on the lower parallel (lines or dots) and their number (one or two) showed that the type of element was significant $[F(1,11)=11.78, p<.01]$, whereas their number was not $[F(1,11)=1.43, p>.05]$. The interaction factor was not significant $[F(1,11)=.27, p>.05]$.

Thus, significant underestimations of vertical extent between oblique parallels occurred in all figures shown in Figure 4 and were greater with dots on the parallels than

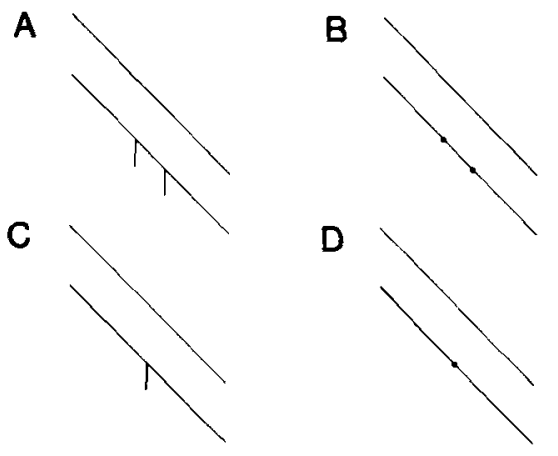

Figure 4. The four stimulus figures for Experiments 1 and 2. Only D was used in Experiment 3.
Table 1

Means and Standard Deviations of Errors in Estimates of Vertical Extent (mm) in Experiment 1 and Errors in Estimates of Alignment or Bisection (mm) in Experiment 2 for the Four Figures in Figure 4

\begin{tabular}{|c|c|c|c|c|}
\hline \multirow{2}{*}{$\begin{array}{l}\text { Stimulus } \\
\text { Figure }\end{array}$} & \multicolumn{2}{|c|}{ Experiment 1} & \multicolumn{2}{|c|}{ Experiment 2} \\
\hline & $M$ & $S D$ & $M$ & $S D$ \\
\hline A & -2.97 & 1.56 & 3.06 & .90 \\
\hline B & -3.76 & 1.65 & 3.63 & 1.43 \\
\hline $\mathrm{C}$ & -2.70 & 1.46 & 1.54 & .54 \\
\hline D & -3.72 & 1.83 & 1.58 & .94 \\
\hline
\end{tabular}

with lines intersecting them. These results confirm those of Greist-Bousquet and Schiffman (1981a) for estimates of oblique extent between vertical parallels. They also found that underestimations were greater with dots than with lines.

\section{EXPERIMENT 2}

The purpose of the second experiment was to determine apparent vertical alignment and bisection in the same oblique parallel-line figure as that in Experiment 1. Subjects were required to make a dot on the upper parallel so that it appeared to be in the same vertical axis as a dot or line on the lower parallel or in the axis of vertical bisection of the space between the pair of dots or lines on the lower parallel.

\section{Method}

There were 12 subjects, 6 men and 6 women, none of whom had participated in the first experiment.

The parallel-line figures (Figure 4) were the same as those in the first experiment. The control figure consisted simply of a single dot in the same position on the response sheet as that in Figure 4D.

\section{Results and Discussion}

The mean score for the single-dot control was $-.34 \mathrm{~mm}$ ( $S D$.45). Since this mean proved to be significantly different from zero $[t(11)=2.63, p<.02]$, the individual scores for the control were subtracted from those for the four oblique-line figures. The resulting mean scores are shown with their standard deviations in Table 1. All four means were positive, that is, in the direction expected for the Poggendorff effect. All 48 individual means were also positive. It can be seen from Table 1 that the means for the two figures with two elements on the lower parallel were greater than for those with a single element, suggesting that displacement errors were greater for the bisection task than for the alignment task. Single-sample $t$ tests showed that the four means were significantly different from zero ( $p<.001$ in all cases). A $2 \times 2$ ANOVA in which the two factors were again the type of elements on the lower parallel showed that whereas the type of element was not significant $[F(1,11)=2.04$, $p>.05]$, the number was $[F(1,11)=49.62, p<.01]$. The interaction was significant $[F(1,11)=24.98, p<$ $.001]$.

In brief, although the type of element on the lower parallel had no effect on the size of displacement of the 
upper dot from a vertical axis, the number of elements did. This outcome is the reverse of that for the estimation of vertical extent. It can be noted also that the greater displacement with two elements on the lower parallel (the bisection task) than with one (the alignment task) accords with the results of the earlier experiment reported by Day and Kasperczyk (1985).

\section{EXPERIMENT 3}

As pointed out above, apparent reduction in extent between the aligned elements in the parallel-line figure cannot by itself account for their apparent misalignment. Apparent reduction would be expected to occur in other directions between the parallels, including that at right angles to them. This expectation is explicit in GreistBousquet and Schiffman's (1981a, 1985, 1986) proposal, in which figures like those in Figures $2 \mathrm{~A}$ and $2 \mathrm{~B}$ were used by way of illustration. In other words, underestimation of extent in the axis of the aligned elements can serve only as an index of a more general underestimation between the parallels. The purpose of the third experiment was to establish whether this is indeed so. Subjects were required to estimate extent between the oblique parallels in the vertical axis, that is, obliquely between the parallels as earlier, and in the right-angle axis, that is, orthogonally between the parallels, in Figure 4D. It could reasonably be supposed that the relationship between apparent vertical and right-angle extent in this figure would obtain for the other figures in Figure 4. Since interest centered on the comparison between apparent extent in the two interparallel directions, control figures consisting of two dots were not considered to be necessary. In any case, the data from Experiment 1 showed that estimates of vertical extent with a two-dot control are more or less veridical.

\section{Method}

A group of 12 subjects who had not participated earlier were tested. There were 8 men and 4 women.

The stimulus figure is shown in Figure 4B. The subjects were required to estimate vertical and right-angle extent between the dot on the lower parallel and a point on the upper parallel. Four estimates of each extent were made with a different random order of the eight estimates for each subject.

\section{Results and Discussion}

All 12 scores for vertical (V) extent were negative, with a mean of $-3.75 \mathrm{~mm}(S D=1.45)$, indicating underestimation. Of the 12 scores for right-angle (RA) extent, 9 were positive, 1 zero, and 2 negative, with a mean of $+1.58 \mathrm{~mm}$ (1.46), indicating overestimation. Singlesample $t$ tests showed that both means were significantly different from zero $\left[t_{\mathrm{V}}(11)=8.95, p<.0001 ; t_{\mathrm{RA}}(11)\right.$ $=3.75, p<.001]$. The difference between the two scores was also significant $[t(11)=16.69, p<.0001]$. Thus, while vertical extent between oblique parallels was underestimated, thereby confirming the results of GreistBousquet and Schiffman (1981a) and Experiment 1, right- angle extent was overestimated. This outcome does not accord with expectations from the explanation of apparent misalignment and misbisection in terms of reduced apparent extent between parallel lines.

\section{EXPERIMENT 4}

The figures in which interparallel extents were estimated in Experiments 1 and 3 differed from the conventional Poggendorff figure in three ways: the parallels were tilted at $45^{\circ}$ rather than vertical, the ends of the parallels were offset rather than aligned at right angles to the parallels, and there were one or two transversals or dots on a single parallel rather than one transversal intersecting each. The question therefore arises as to whether under- and overestimation of extent obliquely and at right angles between the parallels occurs in the conventional figure in which apparent misalignment has been most commonly demonstrated. The purpose of the first experiment was to answer this question. Estimates of both oblique and right-angle extent were made in two figures, one with the ends of the vertical parallels offset from the horizontal (Figure 5A) and one with the ends horizontally aligned (Figure 5B). The reason for including both figures was the possibility that the offset ends could have resulted in a difference in the apparent relative distance of the parallels with consequences for the apparent extent between them.

\section{Method}

A new group of 12 subjects, consisting of 8 women and 4 men, participated.

The subjects estimated extents obliquely between the parallels (intersection to intersection) and at right angles between them (left intersection to right parallel) in the two figures shown in Figures $5 \mathrm{~A}$ and $5 \mathrm{~B}$ and in two two-dot control figures. The dots in the latter were oriented in the same way and were the same distance apart as the designated points on the parallels. The dimensions of the figures were the same as before. In Figure 5A, the ends of the parallels were offset from the horizontal; in Figure 5B, they were horizontally aligned.

\section{Results and Discussion}

The mean scores for the oblique and right-angle twodot controls were $-.67 \mathrm{~mm}(S D .96)$ and $.26 \mathrm{~mm}(S D$ .70). The first proved to be significantly different from zero $[t(11)=2.41, p<.025]$, and the second not so $[t(11)=1.30, p>.10]$. Consequently, the individual scores for the oblique control were subtracted from those for oblique estimates in the parallel-line figures. Those for the right-angle control were not subtracted from the right-angle scores for the two figures. The resultant mean scores for the two parallel-line figures of Experiment 4 are shown with their standard deviations in Table 2. It can be seen that although the mean scores are small, as before, those for oblique $(0)$ extent are negative and those for right-angle (RA) extent, positive. Four independent one-tailed $t$ tests showed that none of the means was significantly different from zero effect $\left[t_{\mathrm{A} . \mathrm{O}}(11)=1.45\right.$, 
A

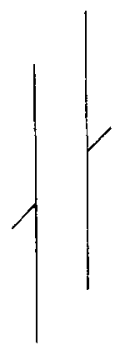

B

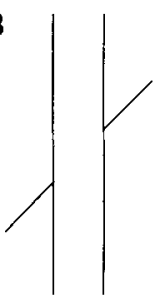

C
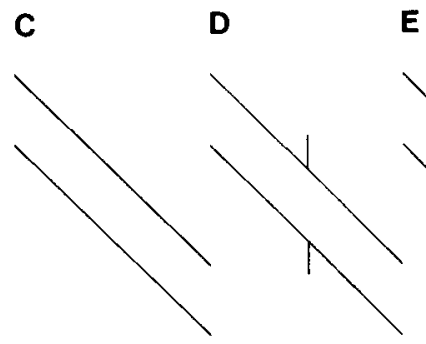

E

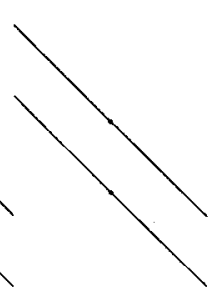

Figure 5. The stimulus figures for Experiments 4 ( $A$ and $B$ ) and $5(C, D$, and $E)$.

$p>.91 ; t_{\mathrm{A} . \mathrm{RA}}(11)=.76, p>.76 ; t_{\mathrm{B} . \mathrm{o}}(11)=.85$, $p>.79 ; t_{\mathrm{B} . \mathrm{RA}}(11)=.52, p>.69$ ]. That is, oblique extents between the parallels were estimated more or less accurately. Furthermore, as can be seen in Table 2, the estimates were more or less the same for the two versions of the figure.

While these outcomes raise the question as to why estimates of extent in the conventional Poggendorff figure and in those versions of it in Figure 4 were different (see below), they also clearly fail to support an explanation of apparent misalignment in terms of reduction in apparent extent between the parallels.

\section{EXPERIMENT 5}

Experiments 1 and 3 were consistent in showing that when the parallels were oblique apparent oblique extent

Table 2

Means and Standard Deviations of Errors in Estimates of Oblique (O) and Right-Angle (RA) Extent (mm) in Experiment 4 for Figures 5A and 5B and of Vertical Extent in Experiment 5 for Figures 5C, 5D, and $5 \mathrm{E}$

\begin{tabular}{|c|c|c|c|}
\hline $\begin{array}{c}\text { Stimulus } \\
\text { Figures }\end{array}$ & $\begin{array}{c}\text { Direction } \\
\text { of } \\
\text { Extent }\end{array}$ & Mean & $\begin{array}{l}\text { Standard } \\
\text { Deviation }\end{array}$ \\
\hline \multicolumn{4}{|c|}{ Experiment 4} \\
\hline A & $\begin{array}{c}\mathrm{O} \\
\mathrm{RA} \\
\mathrm{O} \\
\mathrm{RA}\end{array}$ & $\begin{array}{l}-.73 \\
+.22 \\
-.43 \\
+.16\end{array}$ & $\begin{array}{l}1.73 \\
1.04 \\
1.74 \\
1.04\end{array}$ \\
\hline \multicolumn{4}{|c|}{ Experiment 5} \\
\hline $\begin{array}{l}C \\
D \\
E\end{array}$ & & $\begin{array}{l}-2.50 \\
-0.66 \\
-1.73 \\
\end{array}$ & $\begin{array}{l}1.78 \\
1.81 \\
1.07 \\
\end{array}$ \\
\hline
\end{tabular}

(i.e., vertically) between them was underestimated. Experiment 3 also showed that right-angle extent was overestimated. When, in Experiment 4, the parallels were vertical, both oblique $\left(45^{\circ}\right)$ and right-angle (horizontal) extents were estimated more or less veridically. A possible basis for this difference is suggested by the results for figures with and without a single transversal (Figures 4C and 4D) in Experiment 1. Underestimations of oblique extent were smaller in the former $(-2.70 \mathrm{~mm})$ than in the latter $(-3.72 \mathrm{~mm})$. In other words, the presence of the transversal reduced the degree of underestimation.

The apparent length of a line is increased when collinear extensions are added at its ends (Day, 1977; Lewis, 1909; Sanford, 1897). This figure is a limiting case of the Müller-Lyer figure in which the two lines forming the outward-directed arrows are coincident and collinear with the control line or space. It is therefore conceivable that, in Figures 4C, 5A, and 5B, apparent extent obliquely between the parallels is enhanced, thus counteracting any decrease due to other factors (see below) when the transversals are absent. If this were so it would be expected that in figures similar to those in Figure 4, oblique extent between the parallels, that is, vertical extent, would be underestimated to a greater degree in figures without transversals than in figures with them. This expectation was tested in Experiment 5.

\section{Method}

Subjects were required to estimate vertical extents between two vertically arranged dots the same distance apart as the parallels and between the parallels of the three figures shown in Figures 5C, 5D, and $5 E$. The estimates were the distance vertically between the parallels themselves in Figure 5C, that between the transversalparallel intersections in Figure 5D, and that between the centers of the two dots on the parallels in Figure 5E. The dimensions of the dots, parallels, and transversals were the same as in Experiment 1 . The orientation and offset arrangement of the parallels was also the same.

\section{Results and Discussion}

The mean score for the two-dot control was $-.99 \mathrm{~mm}$ (SD 1.39). Since this proved to be significantly different from zero $[t(11)=2.47, p<.025]$, the individual control scores were subtracted from those for the three parallel-line figures. The resulting mean scores are shown with their standard deviations in Table 2 . It can be seen that underestimation vertically between the parallels (i.e., obliquely to the parallels) occurred for all three figures. The underestimation for the figure with intersecting transversals (Figure 5D) was smaller than that for the figure consisting of parallels alone (Figure $5 \mathrm{C}$ ) or parallels with vertically arranged dots (Figure $5 \mathrm{E}$ ). Three independent $t$ tests showed that the mean scores for Figures $5 \mathrm{C}$ and $5 \mathrm{E}$ were significantly different from zero effect $\left[t_{\mathrm{C}}(11)\right.$ $\left.=4.85, p<.005 ; t_{\mathrm{E}}(11)=5.60, p<.0005\right]$, whereas that for Figure 5D was not $\left[t_{\mathrm{D}}(11)=1.25, p>.05\right]$.

The outcomes of this experiment show clearly that the extent vertically between oblique parallels terminating in collinear transversals that intersect the parallels is more or less accurately estimated, whereas the same extent be- 
tween either the parallels alone or between dots located on the parallels are significantly underestimated. Taken together with the results of Experiment 1, in which underestimation in the same figure was reduced with one transversal, and Experiment 4, in which with vertical parallels it was eliminated between two oblique transversals, these data strongly impugn the argument that the Poggendorff misalignment effect is a secondary outcome of underestimation of extent between the parallels. The fact of the matter is that the apparently misaligned transversal lines reduce or eliminate underestimation of extent.

\section{GENERAL DISCUSSION}

The results of the five experiments can be summarized as follows. Underestimation of vertical extent between oblique parallels occurred in all figures in the first experiment, regardless of whether there were one or two elements on the lower parallel or the elements were dots or lines. However, underestimation was greater for figures with dots than for those with lines but did not vary with the number of elements. In the second experiment, displacements from a vertical axis between oblique parallels occurred in the expected direction for the Poggendorff effect in the same figures and were greater with two elements in the lower parallel (bisection task) than with one (alignment task) but did not vary with the type of elements. The third experiment showed that although vertical extent between oblique parallels is underestimated, that at right angles to them is overestimated. Neither under- nor overestimation of extent in the same directions relative to the parallels occurred when the parallels were upright and the oblique extent between them ended in collinear transversals. That is, in the conventional form of the Poggendorff figure, oblique and right-angle extent were estimated more or less accurately. ${ }^{1}$ In the last experiment, underestimation was again confirmed vertically between oblique parallels but was eliminated, as in Experiment 4, when the extent ended in collinear transversals intersecting the parallels.

These outcomes do not support an interpretation of apparent misalignment and misbisection in the Poggendorff figure or its variants in terms of reduced apparent extent between the parallels. This explanation (Greist-Bousquet \& Schiffman, 1981a, 1981b, 1985, 1986) implies that apparent extent is underestimated between the parallels in directions other than that between the aligned or bisectingbisected elements. This is clearly not so; at right angles to the oblique parallels, extent is, in fact, overestimated. In addition, it could also be expected that apparent misalignment and misbisection would covary with apparent extent. This is not so. Displacement errors that served as an index of apparent misalignment and misbisection were greater with two lines or dots on the parallel than with one but were unaffected by whether the elements were lines or dots. However, extent errors between oblique parallels, that is, underestimations, were greater with dots than with lines but were unaffected by whether there were one or two. Since the displacement errors with one and two elements confirm the data reported by Day and Kasperczyk (1985) and underestimation with lines and dots confirmed those of Greist-Bousquet and Schiffman (1981a), these outcomes can be regarded as robust and reliable. The results from Experiments 4 and 5 also seriously question an interpretation of apparent displacement in terms of apparent extent between the parallels. In the conventional figure with vertical parallels and oblique collinear transversals in which the misalignment effect is commonly demonstrated, apparent extent, both obliquely between the intersections and at right angles to the parallels, is more or less accurately estimated. Finally, with oblique parallels and vertical transversals, apparent extent vertically between the latter is also estimated more or less veridically. Thus, the transversals that appear misaligned in both orientations of the figure serve, in fact, to reduce or eliminate underestimation of apparent extent. In other words, the version of the Müller-Lyer figure in which a line or space terminates in two collinear lines (Day, 1977; Lewis, 1909; Sanford, 1897) eliminates reduced apparent extent between parallel lines.

On this evidence, an explanation of the Poggendorff illusion in terms of reduced extent due to a Müller-Lyer effect between the parallels cannot be sustained. An alternative explanation, also couched in terms of perceptual compromise, is therefore proposed.

Although the notion of a perceptual compromise was originally formulated to explain the Poggendorff effect and its misbisection variant (Tolansky, 1964), it can be extended to a broad range of illusory effects, including illusions of extent between parallels. In parallel-line figures like those in Figures 1 and 5, the distance obliquely between the parallels is less than that at right angles to them. It might therefore be expected that apparent oblique extent would shift slightly toward right-angle extent, and vice versa. In consequence, the former would be slightly underestimated and the latter slightly overestimated. However, both under- and overestimation can be expected to be affected by the introduction of another illusion, such as the Müller-Lyer effect evident in Figures 4C, 5A, 5B, and $5 \mathrm{D}$.

The evidence presented here suggests that two compromises in perception occur in parallel-line figures, one in regard to alignment or bisection and the other in regard to oblique and right-angle extent. The data are clear in showing that these occur independently of each other. Not unexpectedly, underestimation of extent obliquely between parallel lines is eliminated when a version of the MüllerLyer figure formed by collinear lines is added to the parallel-line figure.

\section{REFERENCES}

DAY, R. H. (1977). Perceptual assimilation as a basis for one class of components in geometrical visual illusions. In R. H. Day \& G. V. Stanley (Eds.), Studies in perception (pp. 142-164). Perth: University of Western Australia Press.

Day, R. H., Bellamy, S., \& Norman, A. (1983). On the Morinaga misalignment illusion. Journal of Experimental Psychology: Human Perception \& Performance, 9, 113-125. 
DAY, R. H., \& Jolly, W. J. (1987). A note on apparent displacement of lines and dots on oblique parallels. Perception \& Psychophysics, 41, 187-189.

DAY, R. H. \& KASPERCZYK, R. T. (1985). Apparent displacement of lines and dots in a parallel-line figure: A clue to the basis of the Poggendorff effect. Perception \& Psychophysics, 38, 74-80.

Day, R. H., Watson, W. L., \& Jolly, W. J. (1986). The Poggendorff displacement effect with only three dots. Perception \& Psychophysics, 39, 351-354.

Finlay, D. C., \& CaElli, T. M. (1975). The Poggendorff illusion and estimates of transverse extent. Perceptual \& Motor Skills, 41, 143-148.

Greist-BousqueT, S., \& Schiffman, H. R. (1981a). The Poggendorff illusion: An illusion of apparent extent? Perception, 10, 155-164.

Greist-Bousquet, S., \& Schiffman, H. R. (1981b). The role of structural components in the Mueller-Lyer illusion. Perception \& Psychophysics, 30, 505-511.

GreisT-BousQuet, S., \& Schiffman, H. R. (1985). Poggendorff and Müller-Lyer illusions: Common effects. Perception, 14, 427-447.

Greist-Bousquet, S., \& Schiffman, H. R. (1986). The basis of the Poggendorff effect: An additional clue for Day and Kasperczyk. Perception \& Psychophysics, 39, 447-448.

LEWIS, E. O. (1909). Confluxion and contrast effects in the MüllerLyer illusion. British Journal of Psychology, 2, 21-41.

Morinaga, S., \& IKedA, H. (1965). Paradox in displacement in geometrical illusion and the problem of dimensions: A contribution to the study of perception. Japanese Joumal of Psychology, 36, 231-238.

RESTLE, F. (1976). Morinaga's paradox and figure-ground orientation. Perception \& Psychophysics, 20, 153-156.

SANFORD, E. C. (1897). A course in experimental psychology. London: Heath.
Tolansky, S. (1964). Optical illusions. London: Pergamon.

WARREN, R. M., \& BASHFord, J. A. (1977). Müller-Lyer illusions: Their origin in processes facilitating object recognition. Perception, 6, 615-626.

Wenderoth, P., \& O'Connor, T. (1987). Matching gaps between misaligned pointers demonstrates that lines, both transverse and parallel, enhance Poggendorff illusions. Perception \& Psychophysics, 41, 165-172.

Wenderoth, P., O'ConNor, T., \& Johnson, M. (1986). Evidence for a significant contribution of interactions between oriented line segments in the Tolansky version of the Poggendorff illusion. Perception \& Psychophysics, 39, 334-338.

Wilson, A. E., \& Pressey, A. R. (1976). The role of apparent distance in the Poggendorff illusion. Perception \& Psychophysics, 20 , 309-316.

\section{NOTE}

1. Since it was conceivable that interactions could have occurred between the two forms of the figure to produce the markedly reduced scores in Experiment 4, the experiment was repeated with two new groups of 12 subjects. One group estimated the two extents in Figure 5A, and the other, those in Figure 5B. The outcomes were essentially the same, with no score exceeding $.67 \mathrm{~mm}$ and neither score reaching a reliable difference from zero effect.

(Manuscript received December 9, 1986; revision accepted for publication June 25, 1987.) 